\title{
On Dominance and Context-dependence in Multi-attribute Decisions*
}

\author{
Prasanta K. Pattanaik \\ Department of Economics, University of California, \\ Riverside, CA 92521, U.S.A. \\ E-mail: prasanta.pattanaik@ucr.edu; Telephone: (951) 827 1592; \\ Fax: (951) 8275685 \\ Yongsheng $\mathrm{Xu}$, \\ Department of Economics, Andrew Young School of Policy Studies, \\ Georgia State University, Atlanta, GA 30303, U.S.A. \\ \& \\ Central University of Finance and Economics, China. \\ E-mail: yxu3@gsu.edu; Telephone: (404) 413 0158; \\ Fax: 4044130145
}

This version: 10 August 2008

\begin{abstract}
*Earlier versions of this paper were presented in a conference ("Values and Multidimensional Poverty", 29 May - 1 June 2007) at Oxford Poverty and Human Development Initiative, University of Oxford, and a conference ("Non-welfaristic Welfare: Capability, Choice and Rights", 26-28 October 2007) at University of California, Riverside. We are grateful to the participants in these two conferences for many helpful comments. In particular, we would like to acknowledge our intellectual debt to Salvador Barberà, Kaushik Basu, Rajat Deb, Bhaskar Dutta, Indranil Dutta, Peter Hammond, Mozaffar Qizilbash, and Thomas Schwartz.
\end{abstract}




\section{Introduction}

In this paper, we discuss a difficulty in decision-making when multiple attributes of the

options are relevant for the decision. We start with an informal outline of the difficulty and several examples from various sources in welfare economics, social choice theory, and individual decision making in both ethical and non-ethical matters.

Imagine that you face the problem of choosing between two options, $x$ and $y$. How do you proceed in making your choice? One plausible route will be to identify first what you consider to be the primary or core attributes that you consider relevant for making your choice. Your choice between these two options depends crucially on those attributes identified by you. Typically, you would then commit yourself to the dominance principle with respect to the core attributes that you have already identified, so that, whenever option $x$ is better than option $y$ with respect to some core attributes and no worse than option $y$ in terms of any core attribute, then, overall, you would consider option $x$ to be better than option $y$, and, hence, you would choose $x$ over $y$. If, however, neither $x$ dominates $y$ nor $y$ dominates $x$ (so that, for some core attributes, option $x$ is better than option $y$, and, for some other core attributes, option $y$ fares better than option $x$ ), then you would investigate into the contextual or ancillary features of $x$ and $y$, and your choice will depend on these contextual features: if the contextual features are of one kind, then you would choose $x$ over $y$, and if they are of another kind, then you would choose $y$ over $x$ (in what follows, such dependence on contextual features when neither option dominates the other in terms of the core attributes will be called context-dependence).

If this is how you make your decisions, then your preferences cannot be simultaneously transitive and continuous; this is the basic message that emerges from several examples of decision-making to be found in both economics and philosophy. How can this be? Consider the following informal reasoning. By context-dependence, there are options $x, y, z$ and $w$ such that

(i) $x$ and $z$ are identical in terms of every core attribute, and so are $y$ and $w$, and yet you prefer $x$ to $y$ and $w$ to $z$ because the contextual or ancillary features of $x$ and $y$ are of one kind and those of $z$ and $w$ are of another kind.

(ii) Assume that all core attributes are desirable attributes (for clarificatory comments on this assumption, see Section 3.1). We can then increase the value of some core attribute of $z$ slightly (leaving the values of other core attributes of $z$ unchanged) to get $z^{\prime}$ and decrease the value of some core attribute of $w$ slightly (leaving the values of other core attributes of $w$ unchanged) to get $w^{\prime}$. Since these changes in $z$ and $w$ are assumed to be very small, and you preferred $w$ to $z$ to start with, you will prefer $w^{\prime}$ to $z^{\prime}$ because your preferences over the options are continuous.

(iii) By dominance you must prefer $z^{\prime}$ to $x$ and $y$ to $w^{\prime}$.

(iv) Thus, you prefer $x$ to $y, y$ to $w^{\prime}, w^{\prime}$ to $z^{\prime}$, and $z^{\prime}$ to $x$, so that you have a cycle of strict preferences.

The difficulty presented in the above reasoning is not isolated; it emerges in many decision-making problems in very different areas. We give several examples from the existing literature.

Example 1 (Comparison of living standards). Suppose we have some real indicators of

living standards (or, functionings, to use the terminology of Sen $(1985,1987)$ ), such as health, 
education, nutrition, etc. Using these indicators, all of which represent desirable features, we want to compare the living standards of a given individual in different situations and also the living standards of different individuals. In this context, it has been suggested that, whenever an individual, $i$, is doing better than another individual, $j$, in terms of some of these real indicators and is not doing worse in terms of any one of these indicators, then the overall living standard of $i$ should be deemed to be at least as high as the living standard of $j$, irrespective of the values and/ or the social and cultural backgrounds of the two individuals (the principle of dominance in core attributes, the real indicators being the core attributes). If, however, $i$ is better off than $j$ in terms of some of these indicators and $j$ is better off than $i$ in terms of some of the other indicators, then one can take a 'relativistic' position and allow the values of the two individuals and the norms and mores prevailing in their respective societies to play some role in the comparison of living standards (context-dependence). (Pattanaik and Xu (2007) called such context-dependence in standard of living comparisons "minimal relativism"; the reader may like to refer to their detailed discussion of minimal relativism.) From our earlier reasoning, it then follows that the comparison of living standards cannot be transitive and continuous.

The difficulty is not confined to comparisons of living standards based on real indicators.

Consider the following

Example 2 (The Pareto principle and non-utility considerations). A basic value

judgement, widely used in welfare economics, is the Pareto principle which requires that, if a social state $x$ offers more utility to some individuals in the society as compared to another social state $y$ and no less utility to any individual as compared to $y$, then $x$ is socially better than $y$; this is the principle of dominance in core attributes here, the individuals' utilities being the core attributes. If, however, some individual has higher utility in $x$ than in $y$ and some other individual has higher utility in $y$ than in $x$, then the Pareto principle does not apply. In these cases where the Pareto principle does not apply, it seems tempting to allow non-utility considerations, such as individual rights, freedom, etc., to influence the comparison of the social states under consideration (context-dependence). In such cases, the core attributes are individuals' utilities. It follows that the overall ranking of the social states cannot be transitive and continuous (see Kaplow and Shavell (2001) for a related contribution).

The difficulty goes beyond welfare economics and the theory of social choice. Consider

Example 3 (Individual decision-making in non-ethical matters). Consider an individual

assessing the relative desirability of cars, using several criteria. Whenever a car $x$ dominates another car $y$ in terms of price, durability, and fuel consumption (in the sense that $x$ is better than $y$ in terms of at least one of these three criteria and no worse than $y$ in terms of any of these three criteria), he considers $x$ to be better than $y$ (the principle of dominance in core attributes). If, however, $x$ is better than $y$ in terms of some of the three criteria, and $y$ is better than $x$ in terms of some of the criteria, then the individual allows his ranking to be influenced by his wife's preferences regarding the colour of the cars (context-dependence). In this case, safety, durability, and fuel consumption are the core attributes and the preferences of the individual's wife over the different colours can be regarded as a contextual or ancillary feature. A variant of this example is due to Hare (2007), where an individual assesses cars in terms of two core attributes (comfort and style). If one car is better than the other car with respect to 
both comfort and style, then he ranks the first car higher than the second in an overall comparison of the two cars (dominance in core attributes). If, however, there is a conflict between the rankings of the two cars in terms of comfort and style, then he attaches a greater weight to comfort if the two cars happen to be two different Mercedes (as Hare puts the individual's reasoning here, "comfort is what a Mercedes is really about") but a lower weight to comfort if the two cars happen to be Bentley (as Hare captures the individual's feelings, "style is what a Bentley is really about"). This, of course, is an instance of context-dependence. In such a case, the individual cannot have transitive and continuous preferences over the cars.

And finally, consider

Example 4 (Individual decision-making in ethical matters). This example is again due

to Hare (2007). Consider a situation where an individual is considering what would be morally the right thing to do - to help or not to help a person who is in need of help. There are two considerations which are considered to be core considerations for the moral ranking of the two actions ('help' and 'do not help'): the urgency of the need of the person who needs help and the cost that the decision-maker would incur in providing help . If the decision-maker does not have to incur any cost to help the needy person, then he finds it morally imperative that he should help the needy person (dominance in core attributes). If, however, there is a cost to him of helping the needy person, then his decision depends on the ancillary consideration of whether he knows the needy person and, if so, how well he knows the needy person (context-dependence). In this case, again, the individual cannot have a transitive and continuous preference relation. Note that one of the core attributes here, namely, the cost to the decision-maker, is, intuitively, an undesirable feature for the decision-maker, but, as we explain in Section 3.1, we can formally replace it by a suitably defined desirable feature without losing any part of the underlying intuition.

In all these examples, we face a tension between the principle of dominance in core

attributes, context-dependence, and the requirement that the overall ranking of options should be transitive and continuous. While such tension manifests itself in different ways in these different contexts, there is a common formal structure involved in all these instances. The main purpose of this paper is to propose a general analytical framework in which this common formal structure can be clarified. In the process, we provide general results which imply the results of Kaplow and Shavell (2001), Pattanaik and Xu (2007) and Hare (2007) as special cases. The remainder of the paper is organized as follows. In Section 2, we develop a framework for our analysis. Section 3 introduces several properties of the ranking of the options and presents three of our results. In Section 4, we indicate how our results can be extended. A brief conclusion is given in Section 5 .

\section{The framework}

$U=\{1,2, \ldots, m\}$ is a set of $m$ features or attributes $(\infty>m \geqslant 2)$. The interpretation of these attributes can be different in different contexts. Thus, the utilities that the different individuals enjoy in a social state can be attributes. Similarly, the level of education of a person may be an attribute, and the state of being some particular individual may be an attribute. Note that the notions of "more" and "less" may not make intuitive sense for some of the attributes. Thus, when an attribute refers to the social and cultural background of an individual (see Example 1 in Section 1), the notions of "more" and "less" are not meaningful for the attribute. 
On the other hand, if the attribute refers to a person's education, it makes intuitive sense to talk about more or less of that attribute (i.e., more education or less education).

For every attribute $j$ in $U$, let $X_{j}$ be the set of all values that $j$ can take (\# $X_{j} \geqslant 2$ ). Thus, if the attribute $j$ refers to being some individual belonging to a given society, then $j$ can be any individual in this society and $X_{j}$ can be simply taken to be the set of all the individuals in this society. Let $C$ be a given non-empty proper subset of $U$ satisfying Assumption 1 below. The attributes in $C$ will be referred to as core attributes; the attributes in $U-C$ will be called ancillary attributes.

Assumption 1. For every $j$ in $C$, there exists a linear ordering $R_{j}$ defined over $X_{j}$. Further,

for some attribute $j$ in $C, X_{j}$ is an interval $\left[\beta_{j}, \alpha_{j}\right]$, such that $-\infty \leqslant \beta_{j}<\alpha_{j} \leq \infty$ and for all $s, t \in\left[\beta_{j}, \alpha_{j}\right], s R_{j} t$ iff $s \geqslant t$.

Thus, for every core attribute $j$, it makes sense to speak of more or less of the attribute $j$ (no

such restriction is imposed on ancillary attributes). The linear ordering $R_{j}$ formally captures this notion of more or less. The linear ordering $R_{j}$ stands for the binary relation of "offering at least as much of attribute $j$ as”. For every core attribute $j$ and for all $s, t$ in $X_{j}, s R_{j} t$ denotes that $s$ offers at least as much of attribute $j$ as $t$.

Let $D$ denote the set of those core attributes $j$ for which $X_{j}$ is an interval $\left[\beta_{j}, \alpha_{j}\right]$, such that $-\infty \leq \beta_{j}<\alpha_{j} \leq \infty$ and for all $s, t \in\left[\beta_{j}, \alpha_{j}\right], s R_{j} t$ iff $s \geqslant t$. It may be noted that, given our Assumption 1, the set $D$ is non-empty. Under Assumption 1, it is possible to have core attrbutes (as well as ancillary attributes) which are ordinally measurable but which are not cardinally measurable. Thus, we may be able to distinguish between a higher level of health and a lower level of health, but we may not be able to measure health on a cardinal scale along a real interval.

Several specific frameworks developed in the literature may be noted as special cases of our general framework. In Pattanaik and Xu (2007), $C$ is assumed to be the set of all functionings and all functionings are assumed to be measurable on a ratio scale along a non-degenerate real interval, so that $C=D$ is the set of all functionings. Pattanaik and Xu (2007) assume that the complement of $U,(U-C)$, contains a single element, namely, the attribute of being a specific individual. In Kaplow and Shavell (2001), $C=D$ and the attributes in $D$ denote the utilities of the individuals in a society and $(U-C)$ refers to the set of non-utility considerations.

Let $X \equiv X_{1} \times X_{2} \times \cdots \times X_{m}$ be the set of all conceivable alternatives. Let $\succeq$ be a reflexive binary relation ("at least as good as") defined over $X$. The asymmetric part of $\succeq$ is denoted by $\succ$. Thus, for all $x, y \in X, x \geq y$ is to be interpreted as "alternative $x$ is at least as good as alternative $y$ ", and $x \succ y$ is to be interpreted as " $x$ is better than $y$ ". We say that $\succ$ is acyclic if and only if there does not exist a $\succ$-cylcle in $X$, i.e., there do not exist $x^{1}, x^{2}, \ldots, x^{h} \in X$, such that $\left[x^{1} \succ x^{2}\right.$ and $x^{2} \succ x^{3}$ and ... and $x^{h-1} \succ x^{h}$ and $x^{h} \succ x^{1}$. It is well-known that acyclicity of $\succ$ is a much weaker requirement than transitivity of $\succeq$.

\section{Weak dominance and dominance in core attributes, context-dependence, and weak continuity}




\section{Dominance in core attributes}

As we have noted in our introduction, in many contexts of multicriterial decision-making, it is often assumed that there is a set of core attributes and that, for all alternatives $x$ and $y$ in $X$, if $x$ dominates $y$ in terms of the core attributes (i.e., if $x$ has more of some core attributes and no less of the rest of the core attributes than $y$ ), then, overall, $x$ is at least as good as $y$. The following captures this idea formally.

$\succeq$ satisfies weak dominance (resp.dominance) in core attributes iff there exists $j \in D$ such

that, for all $x$ and $y$ in $X$, if $x_{j}>y_{j}$ and $x_{i}=y_{i}$ for all $i \in C-\{j\}$, then $x \geq y$ (resp. $x \succ y$ ).

Weak dominance and dominance implicitly assume that there is at least one 'desirable'

core attribute that is measured along a real interval. This is true for most of the examples that we discussed in Section 1. What if all the attributes in $D$ are, like the attribute of the decision-maker's cost in Example 4 in Section 1, undesirable? This, however, is not a serious problem. Consider the attribute of cost to the decision-maker. Suppose, this cost can be anything between 0 dollars and 5000 dollars. Then, while modeling the problem, we can replace the attribute of cost by a formally specified attribute $g$ (if one likes, one can call it the decision-maker's monetary benefit), which can take any value in the interval [-5000, 0]. An increase in $g$ will then be intuitively equivalent to a decrease in the cost and will be desirable for the decision-maker. In general, without any loss of intuition, an undesirable attribute, which is measured along a real interval $[\beta, \alpha]$, can be replaced in the formal model by a desirable attribute, which is measured along the real interval $[-\alpha,-\beta]$. A more complex modeling issue, however, arises when, intuitively, none of the core attributes, which are measured along real intervals, is either desirable over the entire range of its permissible values or undesirable over the entire range of its permissible values (so that, starting from some initial quantity of the attribute, an increase in the amount of the attribute is desirable while starting from some other initial quantity, an increase in the amount of the attribute is undesirable). We consider this case in Section 4.

\section{Context-dependence}

Attributes in $C$ are regarded as core criteria for ranking alternatives in $X$. This, however, does not deny the possibility of a role to be played by some ancillary features or attributes in ranking alternatives in some cases. It is possible that, if an alternative, $x$, offers more of some core attributes as compared to another alternative $y$ and $y$ offers more of some of the other core attributes as compared to $x$, then contextual or ancillary considerations may be brought to bear on the comparison between $x$ and $y$. The following two properties capture two different aspects of this idea.

$\succeq$ satisfies context-dependence (type I) iff, there exist $a, b, c, d$ in $X$, such that [for all $j$ in $C$,

$a_{j}=c_{j}$ and $\left.b_{j}=d_{j}\right]$ and $[a \succ b$ and $d \succ c]$.

$\succeq$ satisfies context -dependence (type II) iff, there exist $a$ and $b$ in $X$, such that [for all $j$ in

$\left.C, a_{j}=b_{j}\right]$ and $a \succ b$.

The two properties introduced above specify somewhat differently the notion of

context-dependence. Context-dependence (type I) stipulates the existence of four alternatives 
$a, b, c$, and $d$, such that $a$ and $c$ are indistinguishable in terms of core properties and so are $b$ and $d$, and yet $a$ is ranked higher than $b$ and $d$ is ranked higher than $c$, presumably because of the values of ancillary attributes figuring in $a, b, c$, and $d$, respectively. On the other hand, context-dependence (type II) requires the existence of at least two alternatives $a$ and $b$, such that $a$ and $b$ are indistinguishable in terms of the core attributes and yet $a$ is ranked higher than $b$, presumably because of the differences between $a$ and $b$ so far as the ancillary attributes are concerned. It can be checked that context-dependence (type II) implies context-dependence (type I).

\section{Weak continuity in attributes in $D$}

We now introduce a weak property of continuity of $\succeq$ in attributes in $D$ (recall that every attribute in $D$ is measured along a real interval).

$\succeq$ is weakly continuous in attributes in D iff, for all $x$ and $y$ in $X$ and all $j \in D,([x>y$ and

$x_{j}>\beta_{j}$ ] implies [ for some $x^{\prime}$ in $X, x_{j}^{\prime}<x_{j}, x_{k}=x_{k}^{\prime}$ for all $k \in U-\{j\}$, and $x^{\prime} \succ y$ ]) and ([x>y and $\left.y_{j}<\alpha_{j}\right]$ implies [for some $y^{\prime}$ in $X, y_{j}^{\prime}>y_{j}, y_{k}^{\prime}=y_{k}$ for all $k \in U-\{j\}$, and $\left.\left.x \succ y^{\prime}\right]\right)$.

Weak continuity of $\succeq$ in attributes in $D$ is weaker than continuity (defined in the usual

fashion) of $\succeq$ in the attributes in $D$. To see this, assume that every attribute $j$ can be measured along a real interval $\left[\beta_{j}, \alpha_{j}\right]$ and consider a lexicographic ordering $\succeq$ of the alternatives, such that every core attribute receives priority over every ancillary attribute in the lexicographic ordering. In this case, $\geq$ satisfies weak continuity in the attributes in $D$ (note that $D=C$ here) but not continuity in the attributes in $D$.

\section{Impossibility results}

We now investigate implications of combining weak dominance or dominance in core attributes, context-dependence, and weak continuity of $\succeq$ in attributes in $D$. The following propositions constitute our main findings.

Proposition 1. No transitive $\succeq$ can simultaneously satisfy weak dominance in core

attributes, context-dependence (type I), and weak continuity in attributes in $D$.

The formal proof of Proposition 1 is given in Appendix A. The basic structure of the proof

can, however, be outlined here, using a special case. Let $C=D$ and let $C$ contain exactly two attributes and let both these attributes be 'desirable' in the sense explained earlier in Section 3.1 (in the formal proof, these restrictive assumptions are not needed). Given context-dependence (type I), let $a, b, c$, and $d$ be such that $a_{1}=c_{1}, a_{2}=c_{2}, b_{1}=d_{1}, b_{2}=d_{2}$, and $\left(a \succ b\right.$ and $d \succ c$ ). Given this, it is clear that [for some attribute $j$ outside $C, a_{j} \neq c_{j}$ ] and [for some attribute $j$ outside $C, b_{j} \neq d_{j}$ ]. For the sake of simplicity, assume that for all $j \in\{1,2\}$, each of $a_{j}, b_{j}, c_{j}$, and $d_{j}$ is greater than $\beta_{j}$ (note that this assumption is not required for the formal proof which we give in Appendix $A$ ). Then, by weak continuity of $\succeq$ in attributes in $D$, for some $a^{\prime}$, which has less of attributes 1 and 2 than $a$ and $c$, and some $d^{\prime}$, which has less of attributes 1 and 2 than $b$ and $d$, we must have $a^{\prime} \succ b$ and $d^{\prime} \succ c$. But then, by weak dominance in core attributes, $c \succeq a^{\prime}$ and $b \succeq d^{\prime}$. Together, we have ( $a^{\prime} \succ b, b \succeq d^{\prime}$, $d^{\prime} \succ c$ and $c \succeq a^{\prime}$ ), which contradicts the transitivity of $\succeq$.

Proposition 1 requires $\succeq$ to be transitive. As the next proposition shows, transitivity of $\succeq$ 
can be weakened to acyclicity of $\succ$ if weak dominance in core attributes is strengthened to dominance in core attributes; while dominance in core attributes is stronger than the corresponding weak dominance property, intuitively it is hardly less plausible than its weaker counterpart.

Proposition 2. No $\succeq$ such that $\succ$ is acyclic can simultaneously satisfy dominance in core

attributes, context-dependence (type I), and weak continuity in attributes in $D$.

The proof of Proposition 2 is similar to that of Proposition 1, and we therefore omit it. We

note that, in the above illustration of Proposition 1, if dominance is used for $b$ and $d^{\prime}$ and for $c$ and $a^{\prime}$, then we will get $b \succ d^{\prime}$ and $c \succ a^{\prime}$. Together with $a^{\prime} \succ b$ and $d^{\prime} \succ c$, a cyle is obtained which contradicts the acyclicity of $\succ$.

Proposition 3. No $\succeq$ can simultaneously satisfy weak dominance in core attributes,

context-dependence (type II), and weak continuity in attributes in $D$.

It may be noted that no rationality property is required in Proposition 3. We give the formal

proof of Proposition 3 in Appendix A. We outline the structure of the proof here, again using a special case. Let $C=D$ and let $C$ contain exactly two attributes, attribute 1 and attribute 2, and let both these attributes be desirable in the relevant sense. Given context-dependence (type II), let $a$ and $b$ be such that $a_{1}=b_{1}, a_{2}=b_{2}$, and $a \succ b$. Given this, it is obvious that, for some attribute $j$ outside $C, a_{j} \neq b_{j}$. For the sake of simplicity, assume that $a_{1}=b_{1}>\beta_{1}$ and $a_{2}=b_{2}>\beta_{2}$ (we do not need this assumption for the formal proof). Weak continuity of $\succeq$ in attributes in $D$ implies that, for some $a^{\prime}$, which has less of attributes 1 and 2 than $a$ and $b$, we must have $a^{\prime} \succ b$. Then, by weak dominance in core attributes, we obtain $b \succeq a^{\prime}$, an immediate contradiction.

It can be checked that none of the three propositions above is logically strictly stronger

than any of the other two propositions. It may also be noted that Pattanaik and Xu's (2007) as well as Hare's (2007) prior results are special cases of our Proposition 1. Similarly, the result of Kaplow and Shavell (2001) follows as a special case from our Proposition 3.

\section{Further on weak dominance and dominance}

Though weak dominance in core attributes and dominance in core attributes are plausible properties, they have one rerstrictive feature. They are based on the implicit assumption that, in our intuitive description of the problem, either there exists a core attribute, which is measured along a real interval and which is desirable over the entire range of its values, or there exists a core attribute, which is measured along a real interval and which is undesirable over the entire range of its values so that it can be replaced by a suitably defined core attribute which is desirable over the entire range of its values. While this is true of all the examples that we discussed in Section 1 and we do not know of any example in the existing literature that violates this intuitive assumption, one can nevertheless think of examples where this may not be true. Suppose we have an analytical framework where the well-being of a person depends 
on the level of her calorie consumption, among other things, and calorie consumption is the only attribute in $D$. It is possible that, intuitively, an increase in the level of calorie consumption is considered desirable until it reaches the level of 2500 calories, but, any further increase beyond that is undesirable. In this case, weak dominance and dominance in core attributes, as we have defined these properties earlier, would not be plausible. It is, however, possible to weaken the properties of weak dominance and dominance in core attributes to permit such cases. Consider the following weaker versions of the two properties.

$\succeq$ satisfies local weak-monotonicity iff, there exists $j \in D$, such that, for all $x$ in $X$, there

exists $\epsilon>0$ such that

$x_{j}<\alpha_{j}$ implies either $\left[y \succeq x\right.$ for all $y \in X$ with $x_{j}<y_{j}<x_{j}+\epsilon$ and $y_{k}=x_{k}$ for all $k \in C-\{j\}]$, or $\left[x \geq y\right.$ for all $y \in X$ with $x_{j}<y_{j}<x_{j}+\epsilon$ and $y_{k}=x_{k}$ for all $\left.k \in C-\{j\}\right]$, and

$x_{j}>\beta_{j}$ implies $\left[y \geq x\right.$ for all $y \in X$ with $x_{j}-\epsilon<y_{j}<x_{j}$ and $y_{k}=x_{k}$ for all $k \in C-\{j\}]$, or $\left[x \geq y\right.$ for all $y \in X$ with $x_{j}-\epsilon<y_{j}<x_{j}$ and $y_{k}=x_{k}$ for all $\left.k \in C-\{j\}\right]$. $\succeq$ satisfies local monotonicity iff, there exists $j \in D$, such that, for all $x$ in $X$, there exists

$\epsilon>0$ such that

$x_{j}<\alpha_{j}$ implies either $\left[y>x\right.$ for all $y \in X$ with $x_{j}<y_{j}<x_{j}+\epsilon$ and $y_{k}=x_{k}$ for all $k \in C-\{j\}]$, or $\left[x>y\right.$ for all $y \in X$ with $x_{j}<y_{j}<x_{j}+\epsilon$ and $y_{k}=x_{k}$ for all $\left.k \in C-\{j\}\right]$, and

$x_{j}>\beta_{j}$ implies $\left[y>x\right.$ for all $y \in X$ with $x_{j}-\epsilon<y_{j}<x_{j}$ and $y_{k}=x_{k}$ for all $k \in C-\{j\}]$, or $\left[x>y\right.$ for all $y \in X$ with $x_{j}-\epsilon<y_{j}<x_{j}$ and $y_{k}=x_{k}$ for all $\left.k \in C-\{j\}\right]$. Clearly, local weak-monotonicity and local monotonicity do not involve the implicit assumption that every core attribute, which is measured along a real interval, is either desirable over the entire range of its values or undesirable over the entire range of its values. They simply require the existence of a core attribute $j$, such that $j$ is measured along a real interval, and, starting with any $x$ in $X$, either all small increases in the amount of $j$ make the option more attractive or all small increases in the amount of $j$ make the option less attractive; and similarly for small decreases in the amount of $j$.

Given these properties, we remark that the counterparts of Propositions 1, 2, and 3, using local weak-monotonicity and local monotonicity instead of weak dominance and dominance, continue to hold if we use the following (full) continuity of $\succeq$ in attributes in $D$.

$\succeq$ satisfies continuity in attributes in $D$ iff, for all $x$ in $X$ and all $j \in D$, the sets

$$
\begin{aligned}
& \left\{y_{j} \in\left[\beta_{j}, \alpha_{j}\right]: y \in X, y \succ x, y_{i}=x_{i} i \in U-\{j\}\right\} \\
& \left\{y_{j} \in\left[\beta_{j}, \alpha_{j}\right]: y \in X, x \succ y, y_{i}=x_{i} i \in U-\{y\}\right\}
\end{aligned}
$$

are open in $\left[\beta_{j}, \alpha_{j}\right]$.

It may be noted that the above continuity property is the conventionally used continuity

property in the economics literature. With this property in hand, we can prove the following propositions. Their proofs are, in many ways, similar to the proofs of Propositions 1, 2, and 3, and we omit them.

Proposition 1'. No transitive $\succeq$ can simultaneously satisfy local weak-monotonicity, 
context-dependence (type I), and continuity in attributes in $D$.

Proposition $2^{\prime}$. No $\succeq$ such that $\succ$ is acyclic can simultaneously satisfy local monotonicity, context-dependence (Type I), and continuity in attributes in $D$.

Proposition $3^{\prime}$. No $\succeq$ can simultaneously satisfy local weak-monotonicity,

context-dependence (type II), and continuity in attributes in $D$.

\section{Concluding remarks}

In decisions involving multi-attributes, there is a fundamental incompatibility between various dominance principles and principles of context-dependence in the presence of rationality postulates and the continuity requirement. Dominance principles seem to be natural properties in such situations. Principles of context-dependence are quite weak: a rejection of them would suggest that all attributes excepting the core ones are irrelevant in making decisions. Rationality and continuity seem to be widely accepted requirements in decision theory. If we are prepared not to give up any of these properties of making choices, we are bound to face some dilemma. It would be interesting to examine and investigate further when and how some of these properties may be weakened to arrive at consistent decisions.

\section{References}

Hare, C. (2007), "Rationality and the distant needy", Philosophy and Public Affairs, 35, pp. 161-178.

Fleurbaey, M. (2007), "Social choice and the indexing dilemma”, Social Choice and Welfare, 29, pp. 633-648.

Kaplow, L. and S. Shavell, 2001, "Any non-welfarist method of policy assessment violates the Pareto principle", Journal of Political Economy, 109, 281-286.

Pattanaik, P.K. and Y. Xu, 2007, "Minimal relativism, dominance, and standard of living comparisons based on functionings", Oxford Economic Papers, 59, 354-374.

Sen, A. (1985). Commodities and Capabilities. Amsterdam: North-Holland.

Sen, A. (1987). The Standard of Living. Cambridge: Cambridge University Press.

Weymark, J. (2008), "Must one be an ogre to rationally prefer aiding the nearby to the distant needy?” Presidential Address, 9th International Meeting of the Society of Social Choice and Welfare, June 19-22, 2008, Concordia University, Montreal, Canada.

\section{Appendix A}

Proof of Proposition 1. Suppose a transitive $\succeq$ satisfies weak dominance in core attributes, context-dependence (type I), and weak continuity in attributes in $D$. We shall show that this supposition leads to a contradiction.

By weak dominance in core attributes, there exists an attribute $g \in D$, such that,

for all $x, y \in X$, if $x_{j}=y_{j}$ for all $j \in C-\{g\}$ and $x_{g}>y_{g}$, then $x \succeq y$.

Throughout the rest of the proof, we shall treat such $g$ as fixed. 
By context-dependence (type I),

there exist $a, b, c, d \in X$, such that (for all $j \in C, a_{j}=c_{j}$ and $b_{j}=d_{j}$ ) and ( $a \succ b$ and $d \succ c)$.

We consider the following cases:

$a_{g}=c_{g}=\beta_{g}, b_{g}=d_{g}=\beta_{g}$;

$a_{g}=c_{g}=\beta_{g}, b_{g}=d_{g}>\beta_{g}$

$a_{g}=c_{g}>\beta_{g}, b_{g}=d_{g}=\beta_{g}$;

and

$a_{g}=c_{g}>\beta_{g}, b_{g}=d_{g}>\beta_{g}$.

Suppose (3) holds. Then, by weak continuity and $(a \succ b)$, there exists $b^{\prime} \in X$, such

that, $b_{g}^{\prime}>b_{g}=d_{g}$ and $\left(b_{j}^{\prime}=b_{j}\right.$ for all $\left.j \in U-\{g\}\right)$ and $a \succ b^{\prime}$; and by weak continuity and $(d \succ c)$, there exists $c^{\prime} \in X$, such that, $c_{g}^{\prime}>c_{g}=a_{g},\left(c_{j}^{\prime}=c_{j}\right.$ for all $j \in U-\{g\}$ ) and $d \succ c^{\prime}$. By (1), $b^{\prime} \geq d$ follows from $b_{g}^{\prime}>d_{g}$ and $b_{g}^{\prime}=d_{g}$ for all $j \in C-\{g\}$, and $c^{\prime} \succeq a$ follows from $c_{g}^{\prime} \geq a_{g}$ and $c_{j}^{\prime}=a_{j}$ for all $j \in C-\{g\}$. Therefore, we obtain: $a \succ b^{\prime}, b^{\prime} \geq d, d \succ c^{\prime}$ and $c^{\prime} \geq a$, which contradicts the transitivity of $\succeq$.

Suppose (4) holds. Then, by weak continuity and $(d \succ c)$, there exists $d^{\prime} \in X$, such that, $d_{g}^{\prime}<d_{g}=b_{g},\left(d_{j}^{\prime}=d_{j}\right.$ for all $\left.j \in U-\{g\}\right)$ and $d^{\prime}>c$. By weak continuity and $\left(d^{\prime} \succ c\right)$, there exists $c^{\prime} \in X$, such that $c_{g}^{\prime}>c_{g}=a_{g}$ and $\left(c_{j}^{\prime}=c_{j}\right.$ for all $\left.j \in U-\{g\}\right)$ and $d^{\prime} \succ c^{\prime}$. By (1), $b \geq d^{\prime}$ follows from $b_{g}>d_{g}^{\prime}$ and $\left(b_{j}=d_{j}^{\prime}\right.$ for all $j \in C-\{g\}$ ), and $c^{\prime} \succeq a$ follows from $c_{g}^{\prime}>a_{g}$ and $\left(c_{j}^{\prime}=a_{j}\right.$ for all $\left.j \in C-\{g\}\right)$. Consequently, we obtain: $a \succ b, b \succeq d^{\prime}, d^{\prime} \succ c^{\prime}$ and $c^{\prime} \succeq a$, a contradiction of the transitivity of $\succeq$. The proof for the case where (5) holds is similar to the proof for the case where (3) holds, and we omit it.

Suppose (6) holds. Then, by weak continuity and $(a \succ b)$, there exists $a^{\prime} \in X$, such that $a_{g}^{\prime}<a_{g}=c_{g},\left(a_{j}^{\prime}=a_{j}\right.$ for all $\left.j \in C-\{g\}\right)$ and $a^{\prime}>b$; and by weak continuity and $(d \succ c)$, there exists $d^{\prime} \in X$, such that $d_{g}^{\prime}<d_{g}=b_{h},\left(d_{j}^{\prime}=d_{j}\right.$ for all $\left.j \in C-\{g\}\right)$ and $d^{\prime} \succ c$. Noting that $\left(c_{g}>a_{g}^{\prime}\right.$ and $c_{j}=a_{j}^{\prime}$ for all $\left.j \in C-\{g\}\right)$ and $\left(b_{g}>d_{g}^{\prime}\right.$ and $b_{j}=d_{j}^{\prime}$ for all $j \in C-\{g\}$ ), by (1), we obtain $c \geq a^{\prime}$ and $b \geq d^{\prime}$. Therefore, we have: $a^{\prime} \succ b, b \succeq d^{\prime}, d^{\prime} \succ c$ and $c \succeq a^{\prime}$, which contradicts the transitivity of $\succeq$. This completes the proof of Proposition 1.

Proof of Proposition 3. Suppose $\succeq$ satisfies weak dominance in core attributes, context-dependence (type II), and weak continuity in attributes in $D$. We shall show that this leads to a contradiction.

By weak dominance in core attributes, there exists an attribute $g \in D$, such that (1) holds. In the rest of this proof, we treat such $g$ as fixed.

Given context dependence (type II), there exist $a, b \in X$, such that (for all $\left.j \in C, a_{j}=b_{j}\right)$ and $a>b$.

We distinguish three cases: (i) $a_{g}=b_{g}=\beta_{g}$; (ii) $a_{g}=b_{g}=\alpha_{g}$; and (iii) 
$\beta_{g}<a_{g}=b_{g}<\alpha_{g}$. In case (i), by $a>b$ and weak continuity in attributes in $D$, we have $a \succ b^{\prime}$ for some $b^{\prime} \in X$ with $b_{g}^{\prime}>b_{g}=\beta_{g}$ and $b_{j}^{\prime}=b_{j}$ for all $j \in U-\{g\}$. By (1) and noting that $b_{g}^{\prime}>a_{g}=\beta_{g}$ and $b_{j}^{\prime}=a_{j}$ for all $j \in C-\{g\}$, we have $b^{\prime} \succeq a$, an immediate contradiction of $a \succ b^{\prime}$. In case (ii), by $a \succ b$ and weak continuity in attributes in $D$, we have $a^{\prime} \succ b$ for some $a^{\prime} \in X$ with $a_{g}^{\prime}<a_{g}=b_{g}=\alpha_{g}$ and $a_{j}^{\prime}=a_{j}$ for all $j \in U-\{g\}$. By (1), it follows that $b \geq a^{\prime}$, an immediate contradiction of $a^{\prime} \succ b$. In case (iii), by $a \succ b$ and by a straightforward application of weak continuity in attributes in $D$, we have $a^{\prime \prime} \succ b$ for some $a^{\prime \prime} \in X$ with $a_{g}^{\prime \prime}<a_{g}=b_{g}$ and $a_{j}^{\prime \prime}=a_{j}$ for all $j \in U-\{g\}$. Then, by (1), $b \succeq a^{\prime \prime}$, a direct contradiction of $a^{\prime \prime}>b$. 